\title{
Normas Internacionales de Contabilidad
}

\author{
HÉCTOR BURGOS ALARCÓN
}

\section{RESUMEN}

Las Normas Internacionales de Contabilidad (NIC), son un conjunto de normas que reglamenta la información que debe presentarse en los estados financieros de las empresas, así como la forma como esa información debe registrarse para su análisis.

En junio 29 de 1973 nació el IASC- International Accounting Standard Comité (Comité de Normas Internacionales de Contabilidad) y en Londres, la primera sede, se redactan las normas internacionales de contabilidad. Las NIC se han venido adaptando fácilmente a las necesidades de cada país sin interferir en los procedimientos utilizados por cada Estado en el mundo. Las NIC cambiaron su denominación por International Financial Reporting Standard -IFRS, Normas Internacionales de Información Financiera -NIIF. y emitidas por el Internacional Accounting Standard Boards (IASB), basan su utilidad en la presentación de información consolidada en los estados financieros.

En Colombia y en el mundo se ha venido presentando una serie de discusiones acerca de la necesidad de adoptar estándares internacionales de conducta en materia fiscal, monetaria y financiera y estándares contables más acordes con las transacciones y flujos de mercado, mayor disponibilidad y transparencia de la información sobre datos económicos y financieros. A la vez se exige que estos permitan controlar y combatir el lavado de activos, la corrupción y la evasión fiscal; es decir, incrementar la transparencia en toda la información y que ésta sea útil para la efectiva toma de decisiones. Es necesario que nuestros estudiantes conozcan y estén inmersos en estos nuevos cambios, para así poder asesorar adecuadmente a a las empresas; deben crear una infraestructura contable que sea coherente con las exigencias de las entidades extranjeras.

\section{PALABRAS CLAVE}

Formación ambiental, visión ambiental compleja, competencias cognoscitivas, comunicación ambiental
Las Normas Internacionales de Contabilidad (NIC), es un conjunto de normas que reglamenta la información que debe presentarse en los estados financieros de las empresas, y la forma como esa información debe registrarse para efecto de su análisis. Podemos agregar que son normas de calidad, cuyo objetivo es reflejar la esencia económica de las operaciones realizadas por el negocio y presentar una situación razonable de la empresa, en una fecha determinada. Estas normas son emitidas por el International Accounting Standards Board.

\section{ANTECEDENTES}

En Estados Unidos se creó el Accounting Principles Board-APB, anteriormente el Consejo de Principios de Contabilidad, el cual emitió los primeros enunciados que guiaron la forma de presentar la información financiera. Este consejo fue desplazado por cuanto estaba conformado por profesionales que laboraban en entidades financieras, compañías del Estado y privadas así como en empresas industriales, y su participación en la elaboración de las normas beneficiaba a las entidades para las cuales ellos estaban trabajando.

Posteriormente fue creado el Consejo de Normas de Contabilidad Financiera-FASB que, a la fecha, aún se encuentra vigente en Estados Unidos. Esta entidad logró incidir en la profesión contable, ya que emitió un importante número de normas que transformaron la forma de presentar la información en los estados financieros. Algo importante que se observaba en este comité es que los miembros trabajaban para instituciones educativas como profesores y no se les permitía laborar para entidades con fines de lucro.

1 Monografías.com Marlene Analia Aliaga Riquelme. 
A la vez que se efectuaban cambios importantes por parte del Consejo de Normas de Contabilidad Financiera-FASB se crearon organismos y comités como la Asociación Americana de Contabilidad, el Consejo de Normas de Auditoria y el Instituto Americano de Contadores Públicos; se hicieron publicaciones que difundían pautas sobre el ejercicio de la profesión contable como el Boletín de estudios contables.

En éste proceso, y a través del tiempo, han sido muchas las instituciones mundiales que han venido contribuyendo a generar de cambios en la forma de presentar información financiera, así como el mejoramiento de la calidad de los profesionales de la Contaduría Pública, como la Federación Internacional de Contadores Públicos-IFAC, integrada por 114 países, 156 organizaciones profesionales y dos millones quinientos mil contadores.

Con el transcurrir del tiempo, las empresas iniciaron un proceso de comercialización internacional de sus productos y servicios y, por ende, la información contable de sus operaciones. Ya entonces se podía observar que un comerciante que tenía su planta o negocio en Europa realizaba transacciones con empresarios de América. Éste proceso trajo como consecuencia que las personas involucradas en los negocios codificanran diferentes maneras la información de los balances, por cuanto los procedimientos utilizados por cada país son distintos de acuerdo con sus leyes. Por ello hubo necesidad de crear procedimientos uniformes para la presentación de información en los estados financieros sin tener en cuenta la nacionalidad de quien los estuviera analizando.

\section{Héctor Burgos Alarcón}

Director Programa De Contaduría Pública

Contador público de la Universidad

Central - Catedrático, Asesor Financiero, Auditor y Revisor Fiscal de diferentes empresas, especialmente del sector asegurador.

Para contactar al autor:

hburgos@poligran.edu.co
En junio 29 de 1973 nació el International Accounting Standard Comité (Comité de Normas Internacionales de Contabilidad)-IASC, a través de un convenio de organismos profesionales de varios países, entre otros, Canadá, Estados Unidos, Australia, México, Japón, Alemania, Países Bajos, Irlanda e Inglaterra y Londres, la primera sede, en donde se redactaron las Normas Internacionales de Contabilidad -NIC-.

Podemos decir que las NIC han tenido bastante auge por cuanto éstas se han venido adaptando fácilmente a las necesidades de cada país sin interferir en los procedimientos utilizados por cada estado en el mundo.

\section{PROCEDIMIENTO PARA LA ELABORACIÓN DE UNA NORMA INTERNACIONAL DE CONTABILIDAD}

1- El Consejo establece un comité especial, presidido por un representante, que usualmente incluye otros representantes de las organizaciones profesionales contables de, al menos, otros tres países. Estos comités especiales pueden también incluir a representantes de otras organizaciones, de las representadas en el Consejo o en el grupo consultivo, o bien que sean expertos en el tema a tratar.

2- El comité especial identifica y revisa todos los problemas contables asociados con el tema elegido, y considera la aplicación del marco conceptual para la preparación y presentación de estados financieros elaborados por el IASC-International Accounting Standard Comité, para la resolución de tales problemas. El comité especial estudia, asimismo, las normativas y prácticas contables, ya sean nacionales o regionales, existentes en ese momento, considerando los diferentes tratamientos contables que pueden ser apropiados, según las variadas circunstancias. Una vez que ha considerado los problemas implicados en el tema, el comité especial puede enviar un resumen del punto al consejo del comité de normas internacionales de contabilidad.

3- Tras haber recibido los comentarios del consejo sobre el resumen del punto, si los hubiere, el comité especial normalmente prepara y publica un borrador de 
declaración de principios u otro documento de discusión. El propósito de tal borrador es el establecimiento de los principios contables que formarán las bases de la preparación del proyecto de norma; de igual forma se describe cualquier alternativa o solución considerada, así como las razones por las que se recomienda su aceptación o rechazo.

Se invita a las partes interesadas a realizar comentarios, a lo largo de la exposición pública, que es en promedio de tres meses. En los casos de revisión de una norma internacional de contabilidad existente, el consejo puede dar instrucciones al comité especial para que prepare directamente un proyecto norma, sin publicar previamente un borrador de declaración de principios.

4- El comité especial revisa los comentarios recibidos sobre el borrador de declaración de principios y por lo general, acuerda una versión final de la declaración, que se remite al consejo para su aprobación y uso como base para la preparación de un proyecto de norma internacional de contabilidad. Esta versión final de la declaración de principios está disponible para el público que lo solicite, pero no es objeto de una publicación formal.

5- El comité especial prepara un borrador de proyecto de norma para su aprobación por parte del Consejo. Tras su revisión, y contando con la aprobación de al menos las dos terceras partes del consejo, el proyecto de norma es objeto de publicación. Se invita a realizar comentarios a todas las partes interesadas, durante el período de exposición pública, que dura como mínimo un mes y normalmente se extiende entre uno y tres meses.

6-Por último, el Comité Especial revisa los comentarios y prepara un borrador de norma internacional de contabilidad para que sea revisado por el Consejo. Tras la revisión pertinente, y contando con la aprobación de por lo menos los tres cuartos del consejo, se procede a publicar la norma definitiva.

A lo largo de todo el proceso, el Consejo puede decidir si las dificultades de la materia que se está considerando hacer necesaria una consulta adicional, o si pueden resolverse mejor emitiendo, para recibir comentarios, un documento de discusión o un documento de pro- blemas. También puede ser necesario emitir más de un proyecto de norma, antes de aprobar una norma internacional de contabilidad. De forma excepcional, el Consejo puede, en el caso de problemas relativamente menores, no establecer ni operar mediante el nombramiento de un Comité Especial, pero siempre publica un proyecto de normas antes de aprobar una norma definitiva.

Una pregunta que nos hacemos es ¿por qué deben existir normas de contabilidad únicas? Debe ser de esta forma para que el analista, posibles inversionistas y otros usuarios puedan comparar fácilmente los informes financieros de las empresas que realizan operaciones en diferentes países.

Las normas internacionales de contabilidad se deben adecuar a la contabilidad de las empresas, por cuanto se conseguirá una mayor transparencia y comparabilidad de la información que se utilizará en los mercados financieros, lo que implicará una ventaja competitiva para las empresas.

Puesto que este proceso de adaptación afectará a todos los departamentos de una empresa y no solo al de Contabilidad, es necesario preparar los recursos humanos y tecnológicos oportunamente. Todos los funcionarios beben ser conscientes del proceso de adaptación y entender la importancia y el alcance de un cambio fundamental en la forma en como las empresas medirán sus resultados al presentarlos e al mundo. La adaptación a las Normas Internacionales de Contabilidad es un proceso gradual, que tardará varios años en aplicarse totalmente.

\section{CAMBIO DE LAS NIC POR LAS NIIF}

Las NIC cambiaron su denominación por Internacional Financial Reporting Standard-IFRS, que en español se denominan Normas Internacionales de Información Financiera-NIIF. Y emitidas por el International Accounting Standard Boards (IASB), basan su utilidad en la presentación de información consolidada en los Estados Financieros. 


\begin{tabular}{|c|c|c|}
\hline \multicolumn{3}{|c|}{$\begin{array}{c}\text { CUADRO No 1. Marco conceptual de Normas Internacionales } \\
\text { para preparación de Estados Financieros* }\end{array}$} \\
\hline NÚMERO-NIC & DENOMINACIÓN & COMENTARIOS \\
\hline 1 & Presentación de estados financieros & $\begin{array}{l}\text { Aprobada en 1975, reformada en } 1994 \text { y } \\
\text { revisada en } 1997 .\end{array}$ \\
\hline 2 & Inventarios & $\begin{array}{l}\text { Aprobada en 1975, sustituida en } 1993, \\
\text { vigente desde } 1995 .\end{array}$ \\
\hline 3 & Estados financieros consolidados & Sustituida por NIC 27 y NIC 28 \\
\hline 4 & Contabilización de la depreciación & $\begin{array}{l}\text { Aprobada en } 1976 \text {, reformada en } 1994 . \\
\text { Las NIC } 38 \text { y } 16 \text { sustituyen parcialmente } \\
\text { la NIC } 4 .\end{array}$ \\
\hline 5 & $\begin{array}{l}\text { Información a revelarse en los estados } \\
\text { financieros }\end{array}$ & Sustituida por NIC 1 \\
\hline 6 & Respuesta contable a precios cambiantes & Sustituida por NIC 15 \\
\hline 7 & Estado de flujo de efectivo & $\begin{array}{l}\text { Aprobada en 1977, sustituida en } 1992, \\
\text { vigente a partir de } 1994 .\end{array}$ \\
\hline 8 & $\begin{array}{l}\text { Ganancia o pérdida neta del período, } \\
\text { errores fundamentales y cambios en } \\
\text { las políticas contables }\end{array}$ & $\begin{array}{l}\text { Aprobada en 1978, sustituida en } 1993 \\
\text { vigencia } 1995 .\end{array}$ \\
\hline 9 & Costos de investigación y desarrollo & Sustituida por NIC 38 \\
\hline 10 & $\begin{array}{l}\text { Contingencias y hechos ocurridos } \\
\text { después de la fecha del balance }\end{array}$ & $\begin{array}{l}\text { Aprobada en 1977, reformada en } 1994 \text { y } \\
\text { revisada en } 1999\end{array}$ \\
\hline 11 & Contratos de construcción & $\begin{array}{l}\text { Aprobada en 1979, sustituida en } 1993 \\
\text { vigente } 1995 .\end{array}$ \\
\hline 12 & Impuesto sobre la renta & $\begin{array}{l}\text { Aprobada en 1979, reformada en } 1996 \text { y } \\
2000 .\end{array}$ \\
\hline 13 & $\begin{array}{l}\text { Presentación de activos y pasivos } \\
\text { circulantes }\end{array}$ & Sustituida por NIC 1 \\
\hline 14 & Información financiera por segmentos & $\begin{array}{l}\text { Aprobada en 1981, reformada en } 1995 \\
\text { vigencia } 1998 .\end{array}$ \\
\hline 15 & $\begin{array}{l}\text { Información para reflejar efectos en los } \\
\text { cambios de los precios }\end{array}$ & Aprobada en 1981, reformada en 1994. \\
\hline 16 & Propiedades planta y equipo & $\begin{array}{l}\text { Aprobada en 1982, sustituida en } 1993 \text { y } \\
\text { revisiones en } 1997 \text { y } 1998 . \\
\end{array}$ \\
\hline 17 & Arrendamientos & $\begin{array}{l}\text { Aprobada en 1982, reformada en } 1994, \\
\text { sustituida en 1997, vigente } 1998 .\end{array}$ \\
\hline 18 & Ingresos & $\begin{array}{l}\text { Aprobada en 1982, sustituida en 1993, } \\
\text { vigente } 1995 .\end{array}$ \\
\hline 19 & Beneficios a empleados & $\begin{array}{l}\text { Aprobada 1983, reformada 1995, } \\
\text { revisada } 2000 .\end{array}$ \\
\hline 20 & $\begin{array}{l}\text { Contabilización de las subvenciones del } \\
\text { gobierno }\end{array}$ & Aprobada en 1983 y reformada 1994. \\
\hline 21 & $\begin{array}{l}\text { Efecto de las variaciones de la tasa de } \\
\text { cambio de la moneda extranjera }\end{array}$ & $\begin{array}{l}\text { Aprobada en 1983, sustituida 1993, } \\
\text { vigente } 1995 .\end{array}$ \\
\hline 22 & Combinación de negocios & $\begin{array}{l}\text { Aprobada en 1983, sustituida 1993, } \\
\text { vigente } 1995 .\end{array}$ \\
\hline
\end{tabular}

Continúa... 


\begin{tabular}{|c|c|c|}
\hline \multicolumn{3}{|c|}{$\begin{array}{c}\text { CUADRO No 1. Marco conceptual de Normas Internacionales } \\
\text { para preparación de Estados Financieros * }\end{array}$} \\
\hline NÚMERO-NIC & DENOMINACIÓN & COMENTARIOS \\
\hline 23 & Costos por intereses & $\begin{array}{l}\text { Aprobada en 1984, reformada en } 1994 \\
\text { sustituida en 1993, vigente } 1995 .\end{array}$ \\
\hline 24 & $\begin{array}{l}\text { Informaciones a revelar sobre partes } \\
\text { relacionadas }\end{array}$ & Aprobada en 1984, reformada en 1994. \\
\hline 25 & Contabilización de las inversiones & $\begin{array}{l}\text { Aprobada en 1986, reformada en } 1994 . \\
\text { Sustituida por NIC } 39 \text { y } 40 .\end{array}$ \\
\hline 26 & $\begin{array}{l}\text { Contabilización e información financiera } \\
\text { sobre planes de beneficio por retiro }\end{array}$ & Aprobada en 1989, reformada en 1994. \\
\hline 27 & $\begin{array}{l}\text { Estados financieros consolidados y } \\
\text { contabilización de inversiones en } \\
\text { subsidiarias }\end{array}$ & $\begin{array}{l}\text { Aprobada en 1989, reformada en } 1994 \text { y } \\
\text { revisada en } 2000 .\end{array}$ \\
\hline 28 & $\begin{array}{l}\text { Contabilización de inversiones en } \\
\text { empresas asociadas }\end{array}$ & $\begin{array}{l}\text { Aprobada 1989,reformada } 1994 \text { revisada } \\
1998 \text { y } 2000 .\end{array}$ \\
\hline 29 & $\begin{array}{l}\text { Información financiera en economías } \\
\text { hiperinflacionarias }\end{array}$ & Aprobada 1989, reformada 1994. \\
\hline 30 & $\begin{array}{l}\text { Informaciones a revelar en estados } \\
\text { financieros de bancos e instituciones } \\
\text { financieras y similares }\end{array}$ & Aprobada en 1990, reformada 1994. \\
\hline 31 & $\begin{array}{l}\text { Información financiera de los intereses } \\
\text { en negocios conjuntos }\end{array}$ & $\begin{array}{l}\text { Aprobada en 1990, reformada } 1994 \\
\text { revisada en el } 2000 .\end{array}$ \\
\hline 32 & $\begin{array}{l}\text { Instrumentos financieros: Presentación e } \\
\text { información a revelar }\end{array}$ & $\begin{array}{l}\text { Aprobada 1995, vigencia 1996.revisada } \\
2000 .\end{array}$ \\
\hline 33 & Ganancia por acción & Aprobada en 1997, vigencia 1998. \\
\hline 34 & Información financiera intermedia & Aprobada 1998, vigencia 1999. \\
\hline 35 & Operaciones en discontinuación & Aprobada 1998, vigencia 1999. \\
\hline 36 & Deterioro del valor de los activos & Aprobada 1998, vigencia1999. \\
\hline 37 & $\begin{array}{l}\text { Provisiones, activos y pasivos } \\
\text { contingentes }\end{array}$ & Aprobada 1998, vigencia 1999. \\
\hline 38 & Activos intangibles & Aprobada 1998, vigencia 1999. \\
\hline 39 & $\begin{array}{l}\text { Instrumentos financieros } \\
\text { reconocimiento y medición }\end{array}$ & Aprobada 1999, vigencia 2001. \\
\hline 40 & $\begin{array}{l}\text { Inversión inmobiliaria ( Investment } \\
\text { Property) }\end{array}$ & Aprobada 2000, vigencia 200. \\
\hline 41 & Agricultura & Aprobada 2001, vigencia 2003. \\
\hline
\end{tabular}


Las NIIF aparecen como consecuencia del comercio internacional por la globalización de la economía, en la cual intervienen la práctica de importación y exportación de servicios.

El cambio más importante se presenta al pasar de un esquema de preparación y presentación de estados financieros (manteniendo el capital y el reconocimiento de los ingresos) a otro esquema, completamente diferente, de medición y presentación de reportes sobre el desempeño financiero centrado en la utilidad o en los ingresos provenientes de diversos tipos de transacciones.

\section{LAS NORMAS INTERNACIONALES EN COLOMBIA}

En Colombia y en el mundo se ha venido presentando una serie de discusiones acerca de la necesidad de adoptar estándares internacionales de conducta en materia fiscal, monetaria y financiera y estándares contables más acordes con las transacciones y flujos de mercado, mayor disponibilidad y transparencia de la información sobre datos económicos y financieros, a la vez se exige que éstos permitan controlar y combatir el lavado de activos, la corrupción y la evasión fiscal; es decir, desde todo punto de vista se busca incrementar la transparencia en toda la información y que ésta sea útil para la toma efectiva de decisiones.

Actualmente se encuentra el proyecto de ley 165 de 2007 en la Cámara, por el cual el Estado colombiano adopta las Normas Internacionales de Información Financiera para la presentación de informes contables, cuyos ponentes son los senadores Simón Gaviria Muñoz y David Luna Sánchez.

Dicho proyecto propone que todas las empresas que desarrollen cualquier actividad económica en el territorio nacional, deberán adoptar las normas Internacionales de Información Financiera para la presentación de los estados financieros y delega en el Ministerio de Comercio Industria y Turismo el diseño y la implementación de las mismas.

Es importante resaltar que las empresas tendrán una fecha límite para su desarrollo y en el proyecto quedó como máximo el año 2010; de igual forma, aclara éste proyecto que las pequeñas y medianas empresas o aquellas que tengan que presentar información financiera al exterior por operaciones con entidades de otros países, podrán aplicarlas con fecha máxima hasta el año 2012.

\section{CONCLUSIONES}

Teniendo en cuenta las necesidades del país en este mundo globalizado, se hace necesario que nuestros estudiantes conozcan y estén inmersos en estos nuevos cambios, para así poder dar la asesoría adecuada a las empresas.

Además, como todos sabemos, las micro, pequeñas y medianas empresas en Colombia, son la principal fuente de empleo y a través del tiempo se han convertido en factor fundamental de estímulo a la economía y en la disminución de la pobreza; es por esto que deben crearse herramientas que les permitan ser más eficientes, productivas y competitivas no tan solo nacional sino internacionalmente, incrementando a la vez, el interés por las exportaciones.

Por ello, es importante considerar que estas empresas por su contribución al desarrollo económico nacional, y ante la urgencia de ampliar sus actividades comerciales con el mundo exterior, deben crear una infraestructura contable que sea coherente con las exigencias de las entidades extranjeras; la información contable se debe adecuar a la evolución de otros países, es decir debemos pensar en armonizar las normas colombianas con las internacionales. 